\title{
Aproximación dialógica a las necesidades formativas para perfeccionar la práctica de la extensión rural con campesinos en Caldas, Colombia
}

\author{
A dialogical approach to training needs to improve the practice of rural \\ extension with peasants in Caldas, Colombia
}

\author{
Marlon Javier Méndez Sastoque ${ }^{1}$ (B), Evangelina Giraldo Restrepo ${ }^{1}$ (D)
}

'Departamento de Desarollo Rural y Recursos Naturales, Facultad de Ciencias Agropecuarias, Universidad de Caldas (UCALDAS), Manizales, Colombia.E-mails: marlon.mendez@ucaldas.edu.co; evangelina.giraldo@ucaldas.edu.co

\begin{abstract}
Cómo citar: Sastoque, M. J. M., \& Restrepo, E. G. (2022). Aproximación dialógica a las necesidades formativas para perfeccionar la práctica de la extensión rural con campesinos en Caldas, Colombia. Revista de Economia e Sociologia Rural, 60(1), e242488. https://doi.org/10.1590/1806-9479.2021.242488
\end{abstract}

\begin{abstract}
Resumen: Definir en qué capacitar a los extensionistas rurales es una preocupación constante para quienes asumen esta labor formativa. Tarea de la que derivan múltiples propuestas, de cuya elaboración suelen estar ausentes los propios extensionistas rurales, a pesar del conocimiento vivencial que los autoriza para pronunciarse sobre ello. Bajo esta premisa, con la intención de posicionar un tipo de extensión fundada en la acción dialógica entre extensionistas y campesinos, el estudio responde al siguiente cuestionamiento: a lo luz de lo pensado, expuesto y experimentado por los propios extensionistas rurales, en aras de avanzar hacia el perfeccionamiento de su práctica profesional con campesinos, ¿cuáles son hoy sus necesidades formativas? A partir del diálogo con 59 extensionistas que prestan sus servicios a productores campesinos en Caldas, Colombia, recurriendo al análisis de discurso, se identificaron las siguientes categorías formativas: a) formación para la comunicación efectiva, b) formación para la práctica en contexto, y c) formación para la práctica interdisciplinaria e integral. Se concluye que escuchar la voz de los extensionistas rurales, dándoles protagonismo en la definición localizada de sus principales necesidades formativas, es una vía para el posterior diseño de planes de capacitación cada vez más próximos a las exigencias de la realidad afrontada.
\end{abstract}

Palabras-clave: extensión rural, asistencia técnica agropecuaria, capacitación de extensionistas rurales, desarrollo rural.

\begin{abstract}
Defining how to train rural extension agents is a constant concern for those who undertake this educational work. This is an assignment with multiple proposals from the elaboration of which the rural extension agents are usually absent, despite the experiential knowledge that authorizes them to express on the subject. In this perspective, aiming at position a type of extension founded on dialogical action between rural extension agents and peasants, the study responds to the following question: according to what is thought, said, and experienced by the rural extension agents, what are their training needs today to improve their professional practice in peasant contexts? From the speech with 59 rural extension agents who provide services to rural communities in Caldas, Colombia, using discourse analysis, the following training categories were identified: a) training for effective communication, b) training for practice in context and c) training for interdisciplinary and comprehensive practice. It is concluded that listening to the voice of rural extensionists, giving them a leading role in the localized definition of their main training needs, is a way for the subsequent design of training proposals that are ever closer to the demands of the reality faced.
\end{abstract}

Keywords: rural extension, agricultural technical assistance, rural extension training, rural development. 


\section{Introducción}

En la zona de estudio, situación que se extiende al ámbito nacional colombiano, es común escuchar de empleadores, que los profesionales en ciencias agropecuarias (ingenieros agrónomos, médicos veterinarios, zootecnistas, administradores de empresas agropecuarias, ingenieros agroindustriales) y profesionales en otras áreas (trabajo social, principalmente), al terminar sus carreras e ingresar al mercado laboral, saben muy poco sobre extensión rural. Que, aunque tienen una buena formación general, presentan debilidades en lo concerniente a extensión y desarrollo rural, hecho que atenúan su capacidad de acción en dichas áreas específicas. Circunstancia ratificada en el último diagnóstico nacional de ciencia, tecnología e innovación en el sector agropecuario, en el que se relaciona la baja formación y actualización de los extensionistas con la incapacidad para ofrecer un servicio de extensión idóneo y de calidad (Corporación Colombiana de Investigación Agropecuaria, 2015).

Como argumentos que usualmente soportan dichas lecturas, se menciona que, en las universidades y otros centros educativos, ellos reciben una formación técnica (tecnológica o tecnocientífica) aceptable, pero que en "lo social", término con el que usualmente se hace referencia a los conocimientos relacionados con la extensión y el desarrollo rural, se presenta un vacío que ha sido difícil de llenar (Ramos, 2006; Méndez, 2008). En esta misma línea, algunos autores arguyen que dicha formación, incluyendo la específicamente centrada en extensión rural, se basa en la enseñanza a los futuros profesionales de herramientas y técnicas de comunicación concebidas en el marco de una extensión rural transferencista, técnica y vertical, que mínimamente articula otras dimensiones de la realidad social rural distintas de la técnico-productiva (Santos \& Ramírez, 2016; Lettelier et al., 2016).

Acoplado a lo anterior, también suele argumentarse que la formación sociohumanístico en las facultades de ciencias agropecuarias y afines, área en la que se acostumbra a incluir la extensión rural, ocupa un lugar terciario o secundario, desde una perspectiva que jerárquicamente la ubica por debajo de la formación técnico-científica. En este sentido, estudios develan que el componente sociohumanístico, a pesar de estar integrado a las propuestas curriculares de formación en ciencias agropecuarias, es generalmente subvalorado por los propios estudiantes y algunos docentes que refuerzan entre sus alumnos la supremacía del conocimiento técnico-científico en este tipo de programas (Méndez, 2008). Contexto en el que la formación en extensión para el desarrollo rural llega a tornarse marginal, en atención a la escasa importancia otorgada en el marco de la formación agropecuaria integral.

Ante estas carencias formativas, se argumenta que los extensionistas, principalmente profesionales en ciencias agropecuarias, una vez fuera de las aulas tienen que formarse en la marcha, muchas veces chocando con la realidad enfrentada, ahora sí reconociendo el valor de aquello a lo que en su momento otorgaron mínima atención, así como ganando claridad sobre el oficio o la labor de extensionista. En este sentido, cuando entre los profesionales existe la predisposición para el aprendizaje, aprender de la reflexión crítica acerca de su propia práctica cotidiana, llegando con ello a modificarla, ajustarla o perfeccionarla, tomando como inspiración vivencias que los han puesto a prueba, ha sido el camino para la autoformación, en compensación de la insuficiente preparación previa para el ejercicio de la extensión rural (Diesel et al, 2006; Méndez, 2015; Ringuelet et al., 2015).

En este contexto, aunque se oyen varias voces sobre de la problemática esbozada (la formación de los extensionistas), hay una que, existiendo, suele no escucharse o escucharse muy poco: la de los propios extensionistas rurales, profesionales que están cotidianamente en campo, que ejercen el oficio y pueden dar directamente cuenta del problema. Pues, si se trata de advertir sobre su formación, ¿quiénes mejor que ellos para identificar situada y contextualmente cuáles son sus debilidades y necesidades formativas? Si bien hay propuestas de perfiles y competencias profesionales que permiten inferir necesidades de formación (Méndez, 2006; Russo, 2009; Rodríguez et al., 2017), éstas son casi siempre formuladas por expertos o estudiosos de la extensión y el desarrollo rural, aminorando la participación de los protagonistas, es decir, la de los mismos extensionistas rurales. Actores que tendrían mucho que aportar, sobre todo desde la propia reflexión acerca de su labor profesional cotidiana, al diseño de planes de capacitación desde un principio basados en dar respuesta a los resultados de explorar, participativamente, cuáles son sus necesidades formativas (Landini et al., 2013).

En este escenario problemático, contexto mediado por múltiples afirmaciones y lecturas acerca de la formación de estos actores, surge la siguiente inquietud: a la luz de lo pensado, 
dicho y experimentado por los propios extensionistas rurales, en aras de perfeccionar su práctica profesional con campesinos, ¿cuáles son hoy sus necesidades formativas?

Dar situadamente respuesta a esta pregunta fue el objetivo de este estudio, acotando los hallazgos a lo expuesto y vivenciado por los profesionales (ingenieros agrónomos, veterinarios, zootecnistas, trabajadoras sociales, en mayoría) que ofrecen el servicio público de extensión rural en los 27 municipios departamento de Caldas, Colombia, prestación particularmente dirigida a pequeños productores de economía campesina, desde las Unidades Municipales de Asistencia Técnica (UMATA). Como parte de la institucionalidad estatal concebida para promover el desarrollo agrícola y rural en el país, las UMATA tienen como finalidad brindar a los pequeños productores acompañamiento, asesoría y capacitación técnica, en procura de elevar su capacidad agroproductiva y, como resultado, mejorar sus condiciones de vida, propósito acogido por los extensionistas a estas vinculados. Dicho servicio es gratuitamente ofrecido a los pequeños productores locales, actores en su mayoría propietarios de pequeños predios (minifundios menores a 5 hectáreas), dedicados, junto con sus familiares, al cultivo de café, plátano, caña panelera, hortalizas y frutales, así como a la cría bovinos y especies menores (aves y cerdos) a pequeña escala, productos destinados tanto al autoconsumo familiar como a la venta en los mercados locales.

\section{Metodología}

La investigación, de enfoque cualitativo y orientación comunicativa, se fundamentó en el diálogo abierto entre investigadores y entrevistados, quienes en conjunto conformaron una comunidad de diálogo en torno al asunto de interés: las necesidades formativas de los extensionistas rurales para el perfeccionamiento de su ejercicio profesional en contextos socioproductivos campesinos, bajo un enfoque de extensión dialógica.

Siguiendo esta línea, el estudio constituyó un espacio de reflexión crítica alrededor de los conocimientos y habilidades requeridos para posicionar, en la práctica, un tipo de extensión fundada en la acción dialógica y sinérgica entre extensionistas y campesinos. Ejercicio cimentado, según estudios previos (Méndez, 2020), en las siguientes premisas: a) conocer, para actuar en contexto, las particularidades de cada forma de hacer agricultura, en este caso las de la agricultura campesina y las del medio social en la que esta tiene lugar, b) asumir a los miembros de las comunidades campesinas como sujetos pensantes y actuantes, con capacidad propositiva y de intervención frente a sus propios asuntos, c) actuar concertadamente, conviniendo entre las partes (campesinos, extensionistas y otros posibles actores), a partir del diálogo horizontal, hacia dónde y cómo avanzar en pro de alcanzar condiciones ascendentes de desarrollo agrícola y rural, y d) reconocer la coexistencia de distintos sistemas de conocimiento (campesino, profesional, científico, interdisciplinar, entre otros) que, en vez de negarse mutuamente, pueden complementarse y articularse, uniéndolos para elevar el acervo cognitivo disponible para resolver y aprovechar las problemáticas y las oportunidades surgidas en el contexto de la agricultura campesina.

Bajo esta óptica, la entrevista en profundidad, tipo semiestructurada, fue la principal técnica de colección de información. Cada entrevista, más que un interrogatorio, fue un espacio de diálogo y reflexión en torno a la práctica extensionista en contextos socioproductivos campesinos. Alrededor de las premisas anteriormente señaladas, las conversaciones se centraron los siguientes asuntos:

* Temáticas de interés formativo ligadas a las exigencias del medio social en el que ejercen su labor o a cualquier otro factor considerado.

* Requerimientos de formación evidenciados a partir de situaciones específicas experimentadas en el transcurso de la práctica profesional cotidiana.

El universo o población correspondió a la totalidad de asistentes técnicos adscritos a las UMATA de los 27 municipios del departamento de Caldas, región localizada en el centrooccidente de Colombia. Para realizar las entrevistas, los investigadores se desplazaron a los respectivos municipios. El sitio específico de cada encuentro se negoció con cada entrevistado, procurando que fuera un lugar apto para el diálogo libre y espontáneo. Las entrevistas fueron grabadas y transcritas, con previo consentimiento informado. Cada entrevistado autorizó el uso de los textos transcritos en la presentación de resultados. En total se realizaron 59 entrevistas, entre noviembre de 2018 y octubre de 2019. 
Una vez transcritas, los pasos para su análisis fueron: a) lectura repetitiva de los textos (transcripciones), b) categorización de las respuestas en función de las temáticas formativas y situaciones demandantes de formación emergentes, c) construcción de categorías analíticas descriptoras de las necesidades formativas. Con los resultados de esta primera etapa se elaboró un informe preliminar que fue devuelto a los entrevistados para su respectiva lectura crítica, reconstrucción o complementación, en procura de llegar a acuerdos sobre los hallazgos anotados (Gómez \& Elboj, 2001).

Posterior a la realización de este ejercicio, adaptando lo expuesto por Attride (2001), los pasos seguidos para el análisis final de resultados fueron: a) lectura de los textos validados por la comunidad de diálogo, centrando la atención sobre las observaciones realizadas por los entrevistados, b) confirmación y ampliación de temáticas y situaciones recurrentes asociadas a las necesidades formativas, c) revisión y ajuste de las categorías analíticas construidas.

\section{Resultados y discusión}

El tratamiento de la información permitió identificar y construir tres categorías analíticas englobantes, contendoras de situaciones y temáticas que, a la luz de lo expuesto por los extensionistas rurales, dan cuenta de las necesidades formativas para la acción en contextos socioproductivos campesinos: a) formación para la comunicación efectiva, b) formación para la práctica en contexto, y c) formación para la práctica interdisciplinaria (Tabla 1). A continuación son desarrolladas, incluyendo, para cada una, testimonios típicos que soportan la lectura analítica propuesta.

Tabla 1. Relación de las necesidades formativas para la acción en contextos campesinos expuestas por los extensionistas entrevistados

\begin{tabular}{|c|c|c|}
\hline \multirow{2}{*}{ Necesidades formativas/categorías codificadas } & $\begin{array}{l}\text { Frecuencia de } \\
\text { referencias } \\
\end{array}$ & Porcentaje \\
\hline & $\begin{array}{l}\text { (No. de } \\
\text { entrevistas en } \\
\text { la que se hizo } \\
\text { alusión a cada } \\
\text { categoría } \\
\text { codificada) }\end{array}$ & $\begin{array}{c}\text { (\% sobre el } \\
\text { total de } \\
\text { entrevistas) }\end{array}$ \\
\hline Formación para la interacción efectiva & 53 & 90 \\
\hline Ser vistos y acogidos por los productores como aliados & 47 & 80 \\
\hline Usar un lenguaje comprensible por los pequeños productores & 50 & 85 \\
\hline $\begin{array}{l}\text { Persuadir a los productores para que acaten las } \\
\text { recomendaciones realizadas }\end{array}$ & 16 & 27 \\
\hline Formación para la práctica en contexto & 50 & 85 \\
\hline $\begin{array}{l}\text { Conocer las lógicas económicas y productivas de los campesinos } \\
\text { y pequeños productores }\end{array}$ & 46 & 78 \\
\hline Conocer la especificidad de los arreglos productivos campesinos & 43 & 73 \\
\hline Reconocer el valor de las sociedades rurales & 39 & 66 \\
\hline $\begin{array}{l}\text { Reconocer que las sociedades rurales no están compuestas solo } \\
\text { por campesinos }\end{array}$ & 30 & 51 \\
\hline Formación para la práctica interdisciplinaria e integral & 48 & 81 \\
\hline Planificación financiera & 46 & 78 \\
\hline Asociatividad para el desarrollo agrícola y rural & 43 & 73 \\
\hline Producción limpia o agroecológica & 37 & 63 \\
\hline Formulación de proyectos & 36 & 61 \\
\hline
\end{tabular}




\section{Formación para la interacción efectiva: "saber cómo llegar al campesino"}

Reconocer que la extensión rural se ejerce en interacción con otros, en este caso interactuando con pequeños productores, crea una preocupación ya conocida y, en algunos escenarios, proclamada como el propósito principal de la formación en extensión: "saber cómo llegarle al campesino", expresión reiterativa entre los entrevistados (90\%), aunque dotada de variadas significaciones.

Como primera acepción, "saber cómo llegarle al campesino" expresa la preocupación de los extensionistas por ser reconocido por los otros (productores agropecuarios) como aliados (80\%), es decir, como actores que, convencidos de su capacidad profesional, viene a ayudarles a buscar cómo mejorar sus condiciones productivas y de vida, influenciándolos con sus ideas y propuestas de ajuste:

[Una necesidad formativa] es saber cómo llegarle a la gente, es decir, como hacerles entender que nosotros, los profesionales de la UMATA estamos para servirles. Que nuestro trabajo consiste en ayudarlos a mejorar sus condiciones de vida, a mejorar su existencia y capacidad productiva. Que queremos trabajar a su lado para mejorar la vida de los pobladores del campo y generar allí prosperidad, que queremos, con nuestro conocimiento y experiencia, ponernos a su servicio. (Extensionista No. 12).

Analíticamente, cuando el objetivo es influenciar positivamente el comportamiento del otro para que mejore sus condiciones de existencia, a manera de necesidad formativa, "saber cómo llegarle a la gente" implicaría que los extensionistas aprendan a reconocer cuáles son las aspiraciones de sus interlocutores: propósitos (agro)productivos, objetivos de mejoramiento individual y familiar, pretensiones sociocomunitarias, entre otras intenciones a las cuales plegarse. Una vez generada esta habilidad y reconocidas dichas intenciones, "Ilegarles" consistiría, desde una óptica dialógica, en hacerles saber a los productores la disposición que se tiene para coadyuvarles a avanzar hacia esos propósitos, es decir, en mostrarles cómo conjuntamente podrían caminar en esa misma dirección, siendo conscientes de la necesidad de aprender a negociar, objetivos y prioridades entre productores y extensionistas (Méndez, 2020; Landini, 2021). Práctica que, idealmente, debería darse en doble vía, favoreciendo que el productor también tenga la oportunidad de conocer quién es el extensionista, cuáles son sus pretensiones, fortalezas y proyecciones. Necesidad formativa asociada al aprendizaje para la acción en equipo, bajo una óptica en la que, más que trabajar para los productores, los extensionistas trabajarían junto con ellos, uniendo fuerzas, saberes y experiencias alrededor de propósitos comunes.

Mas, en la práctica, ¿qué tanto los extensionistas, sobre todo en los primeros encuentros, asumen la tarea conocer al otro y darse también a conocer de esta forma?

En este caso, aprender a compatibilizar objetivos, a identificar acciones con potencial de ser emprendidas colectivamente y a unir fuerzas tras ello, sería una necesidad formativa complementaria, para lo cual, el conocimiento mutuo arriba esbozado, sumado al diálogo intercultural, resultaría de gran ayuda:

Para mí, saber qué es lo que quiere el otro, qué está haciendo por su propia cuenta, con qué recursos cuenta, cuáles los conocimientos que aplica para alcanzar sus metas productivas, es totalmente importante. Conocer eso, algo que no siempre hacemos, sería importante para definir un plan que tenga en cuenta las capacidades campesinas, así como sus propios objetivos. (Extensionista No. 23).

Como lo expone el testimonio citado, aprender a valorar el conocimiento y la práctica del otro, convencerse de que los productores, actores rurales también poseedores de un conocimiento experto y de una trayectoria agroproductiva de vida, ya están actuando en función de sus propósitos, hace parte de la necesidad formativa conducente a aprender a unir esfuerzos tras propósitos comunes. Así, conocerse mutuamente, influenciar a la contraparte y dejarse influenciar, otorgándole sentido a lo expuesto por el otro y contraargumentado, cuando sea el caso, las ideas emergentes con las que no se está de acuerdo, permitiría llegar 
a pactos y a propuesta de acción a partir del diálogo y el intercambio constructivo (Méndez, 2020). En esta línea, influenciar al otro sería una actividad lícita, al no tener como fin manipular, sino, al contrario, aportar a construir conjuntamente y avanzar tras un proyecto compartido, práctica constructiva que también tendría que aprenderse.

Opuesto a lo anterior, otra acepción de "saber cómo llegarle al campesino" se aproxima más a la intención de persuadirlo (27\%), tendencia ilustrada en el siguiente testimonio:

En muchas ocasiones, cuando estoy atrasado con mis metas, me gustaría saber más sobre cómo llegarle a la gente, sobre cómo convencer a los productores para que acaten la recomendación a mayor velocidad, para que sigan, sin dudar tanto, los protocolos y las recomendaciones dadas. Si el productor no adopta, si el productor no acoge una recomendación, termina uno siendo el responsable, por no a haber sabido venderle bien la idea. (Extensionista No. 38).

En este caso, la necesidad formativa de los profesionales consistiría en aprender de qué manera convencer a los productores a adoptar ciertas prácticas o tecnologías, en aprender a persuadirlos para que asuman determinados compromisos o adopten comportamientos deseados según un plan predeterminado. En la práctica, "saber cómo convencer al productor", bajo la presión de tener que cumplir metas impuestas (número mínimo de productores que adoptaron un producto o tecnología, número mínimo de organizaciones productivas creadas, número mínimo de usuarios en proceso de reconversión productiva, por ejemplo), se convierte en una necesidad. Situación que surge en un contexto institucional que exige este tipo de resultados y evalúa el desempeño de los profesionales a partir de su poder de persuasión: capacidad de convencer o condicionar al otro para que crea en algo, haga o use algo (Barrientos \& Ryan, 2017).

En síntesis, "saber cómo llegarles a los campesinos", desde las dos acepciones arriba esbozadas, demanda aprender a conocerlos en sus facetas de sujetos actuantes con propósitos, necesidades y aspiraciones múltiples. Conocer dichas preocupaciones les permitiría a los extensionistas actuar en consecuencias, concentrando sus esfuerzos en la búsqueda de respuestas y soluciones útiles, surgidas, según el proceder extensionista, participativa o unilateralmente basadas en el parecer profesional

Desde otro ángulo, con independencia del enfoque (impositivo o cooperativo) orientador, otra necesidad recurrentemente expuesta en el marco de "saber cómo llegarle al productor" tiene que ver con el uso del lenguaje: emplear un lenguaje menos técnico que sea más comprensible para el receptor (85\%). Aprender a moderarlo, a adaptar la jerga técnica al tipo de productor, constituye la principal preocupación práctica:

El productor no necesita que yo le de nombres científicos, que le explique las cosas con una estructura científica, con un lenguaje técnico especializado. El necesita palabras más sencillas. [Ante esta situación], dejar de lado los tecnicismos, usar un lenguaje que le permita al productor entender y encontrarles sentido a las recomendaciones, son estrategias que deberíamos aprender. (Extensionista No. 17).

Como se refleja en el anterior testimonio, aprender a compartir información técnica, en un primer momento recurriendo a un lenguaje simple y comprensible, es un elemento clave en los procesos de extensión, que remite a instruirse en cómo hacerlo. Necesidad formativa referida a aprender a construir, junto con los otros, “[...] un lenguaje común sobre el cual se pueda converger [...]" (Borsese \& Esteban, 2005, p. 2). Es decir, aprender a construir un lenguaje comprensible, que en la marcha introduzca, multidireccionalmente, nuevas palabras y conceptos que adquieran sentido y se agreguen al lenguaje que media el diálogo entre las partes, adaptando así la jerga a las exigencias de cada acto comunicativo.

Aprendizaje que a su vez implica tomar conciencia sobre la existencia de otras formas de comunicar no únicamente basadas en el lenguaje hablado. Bajo esta premisa, la necesidad formativa se ampliaría a la apropiación de estrategias didácticas y pedagógicas útiles para para la comunicación efectiva. Para ello, aprender a usar recursos como las analogías 
metafóricas (establecer relaciones de semejanza con otros conceptos o situaciones cotidianas) (Muñoz, 2010), así como estrategias basadas el aprendizaje sensorial (manipular, construir, rehacer, mirar, tocar, olfatear como forma de conocer y aproximarse a lo nuevo) como complemento del lenguaje verbal (Moreno, 2015), haría parte de esta necesidad formativa.

\title{
Formación para la práctica en contexto: "conocer la realidad del productor campesino"
}

Para el $85 \%$ de los entrevistados, "conocer la realidad del productor" es una preocupación que exterioriza la necesidad de ser conscientes, en lo posible con anterioridad, de la realidad social en la cual ejercerán su práctica profesional. Contexto en este caso mayoritariamente coincidente con la realidad socioeconómica y cultural campesina. Bajo esta lectura, conocer las lógicas económicas y productivas de campesinos y pequeños productores, necesidad identificada por el $78 \%$ de los extensionistas, haría parte del aprendizaje requerido para la acción en contexto:

\begin{abstract}
Nosotros, aquí en el municipio, trabajamos con pequeños productores, con productores de economía campesina, productores con recursos limitados. Sin embargo, para lo que a uno lo preparan en la universidad, para lo que siguen preparando a los nuevos profesionales, es para trabajar con grandes productores. Gente que tiene muchos más recursos, que siembra grandes extensiones, que maneja estructuras empresariales de alto nivel, muy diferentes a la de los productores campesinos. (Extensionista No. 9).
\end{abstract}

Como es reconocido por los entrevistados, en la zona de estudio, la práctica extensionista está principalmente dirigida a campesinos, motivo que les sugiere conocer a profundidad sus formas de producción y de vida. No obstante, como tendencia expuesta por la mayoría de ellos, el conocimiento aprehendido por los extensionistas durante su paso por las aulas se aproxima más al de la realidad agroproductiva empresarial, es decir, al contexto productivo de la gran empresa agrícola, ubicando en un segundo o tercer plano lo referido al entorno campesino. Como señalan Menezes \& Miranda (2011), situación coincidente con la del contexto local y regional, es común que en los pregrados en ciencias agropecuarias se privilegie un único "mundo rural", correspondiente al de los grandes emprendimientos productivos, desconsiderando la existencia de "otros mundos rurales", entre los que se incluye la agricultura campesina. Según lo hallado, se trata de un hecho que en la práctica contribuye a que, una vez inician las labores en campo, los extensionistas se enfrenten al llamado "choque con la realidad", confrontación producto del contraste entre sus presaberes y lo expuesto por la realidad que los acoge: conocimientos asociados a la agricultura empresarial no siempre coincidentes con las particularidades de la agricultura familiar campesina.

Definitivamente, a) que las unidades de producción campesina sean al mismo tiempo unidades de consumo, cuyo propósito es la reproducción familiar y no sólo la maximización de ganancias, b) que la producción de la parcela esté dirigida tanto al autoconsumo como al mercado, en diferentes proporciones, según sea el caso, c) que el factor productivo limitante sea la tierra, seguido del capital, y el abundante la fuerza de trabajo, d) que el ingreso familiar sea bajo y provenga no solo de la venta de los productos cultivados sino también de la venta de la fuerza de trabajo (Valderrama \& Mondragón, 1998; Forero, 2003), entre otros factores, marca diferencia en relación con los modelos de agricultura empresarial, realidad que debe ser conocida en aras de una práctica extensionista en contexto, fundada en la existencia de estas particularidades.

Conocer la realidad de productor también significaría tener en cuenta cuál es su escala productiva, con qué recursos económicos dispone, cuáles son sus preferencias tecnológicas, cómo suele manejar sus parcelas y cuáles son sus propuestas y expectativas de mejora. Se trata de un conocimiento alcanzable en la marcha, a partir del diálogo entre las partes, que puede ser aún más comprensivo y ágil si el extensionista previamente posee bases sobre 
economía y cultura campesina, áreas del saber que han de ser exploradas en su pluralidad (diversidad productiva, cultural, económica y ambiental), para la posterior práctica situada.

En esta misma línea, generar habilidad para la apropiación constante de conocimientos y tecnologías específicamente creados para la pequeña producción, bien sean generados en universidades, centros de investigación o partir del ingenio de los propios productores, sería otra necesidad formativa. Necesidad cubierta a partir de la formación profesional para identificar y acceder permanentemente a redes de información e iniciativas (nacionales e internacionales) de innovación para la pequeña producción (De Souza, 2006), así como para conformar redes cooperativas de ubicación y divulgación de soluciones tecnológicas localmente desarrolladas por los propios agricultores $u$ otros actores rurales locales (De Aquino \& Mengel, 2018). Perspectiva que llama a concebir a los propios productores no solo como meros receptores de conocimiento, sino además en su rol de generadores permanentes de tecnologías endógenas.

Desde un ángulo similar, "conocer la realidad del campesino", de acuerdo con lo expuesto por los entrevistados, también demanda aprender a conocer la especificidad de algunos de sus arreglos productivos (73\%):

\begin{abstract}
Aunque ahora mi mirada es distinta, recién llegado como agrónomo de la UMATA, tuve un choque con la realidad. Al visitar las fincas de los productores, encontré que ellos tenían poco de todo mezclado. Yo pensaba para mis adentros: lo que en esta finca tienen es una mescolanza, pues en mi mente todo estaba separado y ordenado por cultivo. Aunque yo sabía que eso era un policultivo, sinceramente al principio no sabía que recomendaciones darle al productor. Por eso vuelvo a decir que para acompañar a los productores campesinos es necesario pensar como campesino, ver las cosas y los sistemas productivos desde su óptica, algo que los profesionales deberíamos aprender a hacer mejor. (Extensionista No. 33).
\end{abstract}

En referencia a la formación para la práctica en contexto, conocer otras formas de hacer agriculturas distintas a la producción empresarial a gran escala constituye una necesidad formativa. En el caso descrito, conocer lo que para el agricultor representa un policultivo, en términos, por ejemplo, de la seguridad alimentaria, la reducción de riegos ambientales y el aprovechamiento de los recursos disponibles en la finca, ayudaría al extensionista a encontrarle más sentido a la opción por él elegida. Ventajas como: a) tener variedad para comer y vender a lo largo del año o mientras el cultivo principal rinde frutos, b) proteger el ingreso ante la significativa caída del precio de un producto único, y c) aumentar las posibilidades ambientales para controlar enemigos naturales, al incentivar el control biológico y la agrodiversidad, entre otras utilidades difícilmente ofrecidas por el monocultivo (Liebman, 1997; Altieri \& Nicholls, 2010), son razones que soportan su elección, que igualmente han de tener sentido para el extensionista que lee dicha la realidad en contexto y actúa en consecuencia.

En esta línea, actuar en contexto sugiere a los extensionistas aprender a ser conscientes de la posible coexistencia, en un mismo medio sociogeográfico, de múltiples agriculturas: orgánica, agroecológica, familiar, campesina, limpia, biodinámica, empresarial, urbana, de precisión, entre otras, incorporando esa diversidad a su quehacer. Condición que a su vez les demanda poseer conocimientos más amplios y capacidad de adaptación a cada formar de hacer agricultura, sobre todo a aquellas que se apartan de la convencional o dominante, así como tolerancia y respeto frente a lo diferente.

Desde otro plano, otra necesidad expuesta por los entrevistados es aprender a reconocer, en lo posible a lo largo del proceso de formación profesional, cuál es el rol de las sociedades campesinas en la sociedad vista como un todo, como vía para su valoración integral (66\%):

Como parte de la formación, me parece muy importante que a uno como profesional le inculcaran el respeto por el campesino, el buen trato hacia a ellos, porque son personas importantes, completamente abiertas, a quienes debemos respetar, dejando de verlos 
como atrasados o ignorantes. Respeto que también ha de tenerse por el campo, por las labores del campo y su importancia. (Extensionista No. 47).

Analíticamente, aprender a reconocer y valorar, tanto en las aulas como en campo, el rol social de las sociedades campesinas, su aporte crucial a la seguridad alimentaria, al cuidado del ambiente y la provisión de bienes y servicios ambientales, a la conservación de la (agro)biodiversidad, al mantenimiento de la estabilidad política y económica del país, y a la conservación de la cultura nacional (Valderrama \& Mondragón, 1998; Santacoloma-Varón, 2015), entre otras contribuciones, es una necesidad formativa en aras de una buena práctica en contexto. En este escenario, otorgarles a sus miembros este valor, superando los estereotipos que comúnmente los descalifican o representan como simples objetos demandantes de asistencia social, contribuiría a una mejor práctica, esta vez basada en su valoración como sujetos aportantes al bienestar de la sociedad en su conjunto (Méndez, 2016).

Relacionado con la diversidad existente al interior de las sociedades rurales, el estudio permitió identificar otra necesidad formativa: aprender que las sociedades rurales no están compuestas solo por campesinos (51\%):

\begin{abstract}
Es apenas cuando se está en campo que como extensionista uno descubre que en la zona rural hay más que agricultores campesinos. En mi trabajo he tenido que aprender a interactuar con los profesores de las escuelas y colegios, con los líderes de las Juntas de Acción Comunal, con los policías que también adelantan labores de concientización ambiental, con integrantes de otras instituciones y de otras dependencias de la misma alcaldía, interesados en asuntos que no son solamente agrícolas, por ejemplo. Ellos nos ven [a los extensionistas] como profesionales que conocen muy bien la zona rural del municipio, por lo que constantemente nos piden ayuda o nos convocan a hacer proyectos juntos. (Extensionista No. 3).
\end{abstract}

En este caso, la necesidad formativa está asociada al reconocimiento de los múltiples actores que ocupan el espacio rural. Reconocer que en dicho espacio no habitan únicamente productores agrícolas, sino también otros sujetos integrados a las dinámicas sociales rurales, a pesar de su aparente obviedad, es una necesidad sentida. Agricultores, profesores, estudiantes, profesionales de la salud, fuerza pública, dirigentes políticos, comerciantes y prestadores de servicios de diversa índole, mas también mujeres, niños y ancianos, entre otros múltiples actores, conviven e interactúan en los escenarios rurales, dándoles vida y dinamismo.

En este escenario, aprender a reconocer el papel de cada actor, así como sus propósitos y relaciones con los otros, permitiría a los extensionistas rurales identificar y proponer, según las circunstancias, posibilidades de acción sinérgica alrededor de asunto claves o de interés común. Diversidad que también ha de tenerse en cuenta para la planificación diferenciada e incluyente. Ser mujer, niño o anciano, entre otros referentes específicos, otorga particularidad a los sujetos rurales, circunstancia a ser considerada en la práctica extensionista integral, en pro de una intervención contextualizada que comprenda, valore e incorpore dichas singularidades.

\title{
Formación para la práctica interdisciplinaria e integral: “la extensión va más allá de la asistencia técnica a cultivos"
}

Como sugiere el estudio, la práctica cotidiana enfrenta a los extensionistas, principalmente a los formados en ciencias agropecuarias, a situación que superan sus conocimientos o experticias iniciales. Según lo corroborado en campo, asuntos problemáticos a resolver, incluyendo algunos de alta repercusión sobre las dinámicas agroproductivas, pueden tener un trasfondo cultural, político o ambiental, condición que demanda intervenirlos recurriendo conocimientos y estrategias más allá de los usualmente utilizados para revolver cuestiones de índole agrotécnica. En este contexto, quienes trabajan directamente con pequeños productores, coincidiendo con lo expuesto por Landini (2015), 
pronto descubren que la complejidad de la realidad enfrentada no puede ser intervenida únicamente con capacidades técnicas, circunstancia de la que deriva la necesidad de formación para la práctica interdisciplinaria:

Cuando se quiere ejecutar una asistencia técnica integral, los programas y proyectos deben considerar todos los aspectos, por lo que requiere integrar equipos con varios profesionales. Uno que sepa de agronomía, otro experto en veterinaria, otro experto en asuntos comunitarios y sociales, otro en economía o comercialización. Para poder avanzar en beneficio de ellos [los productores] se requieren de todas esas disciplinas. Sabemos que su problemática es compleja, pero también sabemos que igualmente es complejo trabajar en equipo, cuando cada uno está acostumbrado a dar cuenta apenas de su especialidad. (Extensionista No. 54).

Como se esboza en el testimonio típico citado, la integralidad en el servicio de extensión, preocupación expuesta por la mayoría de entrevistados (81\%), demanda sumar saberes alrededor de asuntos cuya intervención convoca a más de un saber o disciplina. En este caso, la necesidad formativa consistiría en aprender a actuar interdisciplinariamente o, por lo menos como paso previo, a concebir que algunos de los problemas enfrentados requieren ser intervenidos desde diferentes ángulos. Bajo esta óptica, aprender a establecer objetivos compartidos entre los miembros de un equipo de extensión, a cuyo cumplimiento cada uno aporte desde su experticia y formación disciplinar, sería el camino a seguir:

Lograr el desarrollo rural del municipio requiere actuar, al mismo tiempo, desde lo productivo y lo social. Buscar el desarrollo productivo, es decir, que en el municipio se produzca una parte importante de lo que aquí se consume, debe ir de la mano del desarrollo de la gente: que la gente en el campo esté contenta, que tenga educación, salud, recreación... Sobre esto yo hablo con las trabajadoras sociales, pero también con los veterinarios, agrónomos e ingenieros de alimentos que hacen parte del equipo: ¿cómo hacer para alcanzarlo?, ¿estamos preparados para ello? (Extensionista No. 35).

Proceder de esta forma consistiría en establecer formas de trabajo interprofesional para la resolución colaborativa de problemas, aminorando la excesiva fragmentación de saberes ligada a la alta especialización. En este caso, aprender a generar condiciones de cooperación e intercambio entre actores expertos en diferentes disciplinas (profesionales en ciencias agropecuarias y profesionales formados en ciencias sociales, por ejemplo) sería la clave para llegar a acuerdos sobre cómo enfrentar conjuntamente situaciones problemáticas, incluyendo además la participación de los propios productores.

En esta dinámica, aprender a actuar interdisciplinariamente se cruzaría con aprender a actuar en grupo. Aprendizaje basado en que cada miembro de los equipos de extensión desarrolle habilidades para: a) conocer la experticia de los otros, ganando claridad acerca de sus saberes, pericias, campos de acción, habilidades y destrezas, b) identificar y adoptar como propios los intereses y objetivos que los unen como equipo, y c) generar ambientes de comunicación continua, cooperación y coordinación de actividades en torno a propósitos y metas compartidas (Vizcaino \& Otero, 2007; Gil et al., 2008).

Con todo, aunque el ideal expuesto por los entrevistados es que las unidades locales cuenten con equipos de trabajo interdisciplinarios (agrónomos, veterinarios, zootecnistas trabajando de la mano con profesionales expertos en trabajo social, desarrollo familiar, psicología, agroindustria, administración agropecuaria, ingeniería de alimentos, principalmente), esto no siempre se logra. Limitaciones asociadas a la baja capacidad presupuestal y al desinterés de los gobernantes de turno por el desarrollo rural hacen que profesionales de las ciencias agropecuarias, sin la compañía de profesionales de otras áreas, sean quienes en sus localidades tengan que dar cuenta de múltiples requerimientos sociocomunitarios que van más allá de lo técnico-agroproductivo. Hecho que convoca a fortalecer la formación integral de los profesionales del agro, otorgándole el debido valor al componente sociohumanístico dentro de las propuestas curriculares. 
Pasando a un plano más concreto y vivencial, el análisis de los diálogos permitió identificar cuatro áreas claves de formación a reforzar que, alrededor de un problema particular, convocan o atañen directa o indirectamente a varias ramas del saber. Estas áreas son:

a) Planificación financiera de los predios agropecuarios (78\%). Aunque la formación en el campo económico-administrativo hace parte integral de los planes curriculares de las carreras cursadas por los extensionistas entrevistado, según lo indagado, el foco puesto en lo técnico-productivo hace que se disipe la visión integradora. En este sentido, es común hallar en campo extensionistas con amplia formación en manejo de cultivos y producción animal, aunque con pocos o muy básicos conocimientos en gestión del negocio agropecuario (López, 1998; Sánchez, 2016), asunto que tiene efecto sobre su desempeño:

Como Médico Veterinario Zootecnista, mi fuerte es acompañar al productor para que produzca bien y con calidad. En eso siento que tengo fortalezas. Sin embargo, sé que el proceso productivo no acaba con obtener un buen producto, el producto tiene que ser vendido. Por eso me gustaría tener más bases sobre análisis de mercados, pensando en que el productor pueda ser más competitivo, que no llegue a correr el riesgo de recibir poco por lo que produce. (Extensionista No. 4).

En síntesis, "[...] aplicar principios y procedimientos administrativos para un uso y combinación más eficiente de los recursos productivos de que dispone la finca [...]" (López, 1998, p. 206), con el ánimo de obtener sostenidamente los máximos beneficios económicos y sociales, es la razón de ser de la administración agropecuaria. Sobre esta lectura, la planificación del predio debe considerar, además de un plan agroproductivo, un plan de inversión y uno financiero, siendo el propósito de este último auxiliar al administrador o dueño de la finca en la toma de decisiones que comprometen su capital. Hecho que, en términos de apoyar eficazmente al productor, demanda al extensionista rural poseer formación en planificación financiera.

En este caso, tal como lo sugieren el testimonio típico citado, tener conocimientos sobre el uso de herramientas de análisis financiero, destacando algunas como el estudios de mercados, la cuantificación del capital de trabajo, la determinación de relaciones costobeneficio, el análisis de sensibilidad ante riesgos, el cálculo de puntos de equilibrio, la proyección de tasas de retorno, márgenes de utilidad y rendimientos sobre el capital (Villegas, 2002) sería una necesidad formativa acorde con las reglas del modelo actual modelo económico predominante. Que el extensionista las apropie para luego promover su uso entre los productores sería el camino a tomar, principalmente bajo un enfoque de extensión fundado en impulsar su inserción efectiva a la dinámica de mercado, para lo cual, que el productor adopte una mentalidad calculista sería una prioridad.

Analíticamente, a manera de lectura alternativa, lo dicho por los extensionistas igualmente deja entrever otra necesidad formativa implícita: ganar conciencia acerca de la existencia de otras racionalidades económicas distintas a la hoy predominante. Sin pretender agotar el tema, reconocer que la actividad económica campesina, más que a obtener ganancias sobre el capital invertido, principalmente se orienta a la subsistencia y reproducción social del grupo familiar (Cáceres et al., 1997; Van der Ploeg, 2009; Landini, 2011), que campesinos y pequeños productores, ante la imprevisibilidad de las condiciones agroproductivas, prefieren aminorar los riesgos por encima de maximizar los ingresos (Cáceres et al., 1997; Landini, 2011), entre otros descriptores de la racionalidad campesina, contribuiría a elevar dicho grado de conciencia, para luego actuar en consecuencia, proponiendo esquemas de planificación diferenciales.

En síntesis, la necesidad formativa se concreta en aprender a articular los sabres agroproductivos (fitotécnicos y zootécnicos) con los derivados de las ciencias económicoadministrativas, en procura de un uso más eficiente de los recursos disponibles, según los propósitos socioproductivos trazados. Objetivos que podrían centrarse en maximizar la 
producción y los márgenes de ganancia del negocio agropecuario o, cercano al ámbito de la economía campesina, en proporcionar a las familias mayores grados de seguridad alimentaria y niveles ascendentes de independencia económica.

b) Asociatividad para el desarrollo agrícola y rural (73\%). Actualmente, en Colombia, estar organizados (constituidos como asociación o cooperativa, entre lo más mencionado) es casi una obligación para los pequeños productores que aspiren a recibir apoyos gubernamentales o privados. Situación reconocida por los extensionistas rurales, quienes suelen asumir como tarea común fomentar localmente la asociatividad para el desarrollo agrícola y rural. En este contexto, se toma como punto de partida que estar asociados brinda a los productores algunas ventajas como: a) mayor facilidad de acceso a capacitación, asesoría técnica, y servicios financieros (Ferraro \& Goldstein, 2011), b) cumplimiento, como grupo, de los requerimientos exigidos para establecer alianzas productivas con otros actores locales y regionales, c) mayor probabilidad de acceso a bienes públicos (Machado, 2000), incluyendo la incorporación a programas y proyectos de origen gubernamental, mixto o privado, y c) mayor capacidad de respuesta a las amenazas del entorno (Amézaga et al., 2013) en comparación con la facultad que individualmente tendría cada miembro del grupo.

En este escenario, poseer conocimientos y habilidades para fomentar la creación de nuevas organizaciones y coadyuvar a consolidar las ya existentes emerge como necesidad formativa. Necesidad, según los hallazgos de la investigación, principalmente asociada a resolver problemas intrínsecos a la organización en sí:

Muchas de las organizaciones que existen en el municipio presentan problemas desde su creación. Varias fueron creadas para acceder a Alianzas Productivas, convocatorias a las que solo se puede acceder con productores organizados. Así, ante el afán de las convocatorias, constituir las organizaciones ha sido también un afán. A los productores se les presenta la idea, muchos se animan y deciden agruparse, pero a veces sin saber muy bien para qué, solo pensando en los beneficios que podrán adquirir. Luego, cuando empieza a pasar el tiempo, vienen los problemas con la organización. Algunos se quieren retirar, no quieren cumplir con los compromisos adquiridos, ante lo que nosotros, los extensionistas, tenemos que reaccionar e intervenir, tratando de mediar en la resolución de esos problemas. (Extensionista No. 11).

Definitivamente, enfrentar y vivenciar realidades como las mencionadas alerta a los extensionistas sobre lo difícil que puede llegar a ser la organización como estrategia para el desarrollo agrícola y rural, pese a las ventajas anteriormente esbozadas. Asunto exacerbado cuando las organizaciones han surgido improvisadamente, casi siempre en respuesta a los requerimientos inmediatos de una entidad promotora o financiadora de programas 0 proyectos, circunstancia que logra desgastar el modelo asociativo como opción promisoria para los pequeños productores. Ante esta problemática, aprender a reivindicar la importancia de la organización como estrategia de acción conjunta para alanzar objetivos compartidos sería de la principal necesidad formativa.

En este orden, formarse para apoyar procesos asociativos implicaría, entre otros aspectos, adquirir habilidades para: a) fomentar el liderazgo, la visión de colectivo y la participación activa de los miembros de la organización en la toma concertada de decisiones, b) animar, entre los asociados, la construcción de confianza, a partir del establecimiento de acuerdos sobre los derechos y deberes, los mecanismos para resolver conflictos, los incentivos y las sanciones a lugar, c) impulsar la construcción de una cultura asociativa, promoviendo la vivencia de valores como la solidaridad, la lealtad, la equidad, la transparencia y la honestidad, y d) apoyar la puesta en marcha de mecanismos fluidos de comunicación e información entre los integrantes de la organización (Camacho et al., 2007), acciones en conjunto fortalecedoras de la organización para el desarrollo agrícola y rural.

En resumen, es una necesidad formativa que convoca a la apropiación analítica e instrumental de saberes procedentes de las ciencias sociales (sociología, psicología, 
antropología) en función del fortalecimiento de la organización en torno a asuntos agroproductivos (producción, comercialización, acceso a múltiples recursos), aunque también de otras índoles (política, ambiental, cultura), de acuerdo con las especificidades del caso. En cualquier circunstancia, más allá de lo formal (saber cómo constituir una organización en el papel), aprender a impulsar el liderazgo, la construcción de una cultura asociativa y la vivencia de valores solidarios al interior de las comunidades participes sería parte de la formación interdisciplinaria requerida.

c) Producción limpia o agroecológica (63\%). Para los extensionistas entrevistados, reconocer en campo los problemas ambientales asociados a la agricultura ha sido un aliciente para cuestionar las formas convencionales de producción. La dependencia de insumos agroquímicos, la degradación de los suelos y las fuentes de agua, el uso desmedido de pesticidas para el control de plagas y enfermedades, entre los factores más mencionados, los lleva a preguntarse sobre la existencia de alternativas productivas, así como a cuestionarse sobre su falta de preparación al respecto:

Producir limpiamente, producir de una manera más amigable con el ambiente, es una necesidad actual. Sin embargo, como profesional, es poco lo que sé al respecto. Mis conocimientos están más orientados a la agricultura convencional, es decir, a la agricultura basada en el uso de insumos de síntesis principalmente química: fórmulas de N-P-K y micronutrientes, insecticidas y fungicidas para el control fitosanitario, entre lo más usado, insumos que deberían reemplazarse gradualmente por otros menos nocivos u ecológicos, pensando en el cuidado medioambiental. (Extensionista No. 29).

Lo hallado permite evidenciar que la necesidad expuesta por los entrevistados sigue siendo predominantemente técnica, sintetizada en adquirir capacidades para promover una agricultura menos lesiva al ambiente: sustituir productos agroquímicos tóxicos y costosos por insumos alternativos, como biofertilizantes y biopesticidas más ambientalmente afables (Altieri, 1994). En este contexto, la necesidad formativa estaría enmarcada en un enfoque de agricultura sustentable fundado en el reemplazo de prácticas e insumos ambientalmente nocivos y riesgosos por otros más ambientalmente amigables (Caporal \& Dambrós, 2017). Postura con sentido, aunque insuficiente para dar respuesta a la crisis socioambiental en curso.

En este contexto, cuando el interés está en lo técnico-productivo, combinando lo expuesto por Altieri (1994) y Lacey (2015), las necesidades formativas de los extensionistas rurales podrían centrarse en adquirir conocimientos para:

* Eliminar gradualmente el uso de insumos químicos y moderar su uso en combinación con el manejo integrado de plagas y enfermedades.

* Sustituir insumos agroquímicos por otros de carácter biológico.

* Diseñar sistemas agrícolas que estimulen el sinergismo entre especies animales y vegetales (sistemas agrosilvopastoriles).

* Proteger y conservar los suelos mediante el manejo de cubiertas vegetales y prácticas de labranza que minimicen la erosión.

* Promover el uso de variedades y razas autóctonas y rústicas adaptadas a las condiciones locales, que respondan a un manejo bajo en insumos.

Formarse en líneas como las mencionadas, centradas en el carácter técnico de la práctica agroecológica, respondería al requerimiento declarado por los extensionistas que hicieron parte del estudio. No obstante, es necesario reconocer que una extensión que contribuya a un desarrollo agrícola y rural más integral y sostenible debería atender a otros aspectos más allá del técnico-productivo. Fomentar la equidad social, la estabilidad y sustentabilidad ambiental, la diversidad agrícola, biológica y sociocultural, entre otras acciones amparadas en los postulados del saber agroecológico, haría parte de una práctica extensionista más completa. 
En este sentido, adquirir conocimientos y habilidades para la puesta en marcha de prácticas como las a continuación mencionadas permitiría a los extensionistas, en términos de necesidad formativa, ampliar su visión y capacidad para promover procesos más holísticos de transición agroecológica:

* Reconstrucción de sistemas agrícolas basados en la aplicación del conocimiento tradicional acumulado, estimulando el diálogo y la integración entre éste y el conocimiento derivado de las ciencias convencionales, en procura de sinergias que permitan avanzar hacia una agricultura más sustentable (Delgado \& Rist, 2016; Caporal \& Dambrós, 2017)

* Potenciación del uso de los medios y recursos localmente disponibles, incluyendo elementos asociados a las formas tradicionales de organización e interacción social, política y económica, en el diseño de estrategias de avance hacia la sostenibilidad socioambiental (Caporal \& Dambrós, 2017).

* Desarrollo participativo de propuestas de producción y consumo que contribuyan a enfrentar, transformativamente, la crisis ecológica y social que actualmente enfrenta la sociedad en su conjunto (Norgaard, 1994; Alemany \& Sevilla, 2007).

Se trata de estrategias de naturaleza más sistémica, que dan protagonismo a los propios productores como forjadores de dinámicas sociales orientadas no sólo a transitar hacia la producción técnico-agroecológica sino principalmente hacia el cambio social. Contribuir a fraguar una sociedad más sostenible, basada en principios de equilibrio ecológico, horizontalidad, justicia y equidad social, sería, en este caso, el propósito principal de aprendizaje.

En síntesis, es una necesidad que interdisciplinariamente llama a un diálogo más amplio entre el saber agronómico convencional y la agroecología, en procura de complementariedades que permitan avanzar hacia una agricultura y una sociedad cada vez más sostenibles. En esta línea, apropiar y promocionar la adopción de técnicas de producción limpia derivadas del saber agroecológico, así sean acciones enmarcadas en procesos de extensión convencional (centrados en el cambio tecnológico), sería un avance significativo hacia la sostenibilidad anhelada. Acción multiplicable en su efecto, si lo aprendido en relación con el saber agroecológico fuera más allá de lo técnico-productivo, ampliándose a la trasformación social como respuesta a la problemática ambiental contemporánea.

d) Formulación de proyectos (61\%). Frente un panorama de escases, consistente en que los recursos financieros con los que cuentan los extensionistas para operar e invertir en propuestas locales de desarrollo agrícola y local son mínimos, los entrevistados plantean que aprender a gestionar recursos ante instancias bien sean internas o externas es una necesidad apremiante. Presentar propuestas coherentes, con objetivos claros y de alta pertinencia social, además de ser económica y culturalmente viables, hace parte de sus tareas cotidianas. En esta línea, saber formular proyectos, habilidad que ha de ir acompañada del conocimiento acerca de cuáles son y dónde están las distintas agencias cooperantes o financiadoras, emerge como necesidad formativa. No obstante, cómo los muestran el siguiente testimonio común entre los entrevistado, la preocupación inicialmente expuesta reduce la formulación de proyectos a llenar los formatos dispuestos para ello:

Saber diligenciar los formatos del Ministerio de Agricultura y otras agencias financiadoras, es una necesidad nuestra. Llenarlos bien, escribir muy bien lo que están solicitando, manejar bien la plataforma virtual dispuesta para la formulación de los proyectos es algo clave para el acceso a recurso. (Extensionista No. 56).

En este caso, sin desconocer la importancia de esta habilidad, formular proyectos se asume como una acción casi mecánica, dirigida a seguir guías y diligenciar campos vacíos bien sea sobre el papel o en línea. Acción guiada por una racionalidad instrumental y tecnocrática (Bauman, 1989; Nussbaum, 2011), que hace que el interés de los profesionales se centre más 
en aprender a usar con experticia los medios (guías e instrumentos para la formulación de proyectos), que en el fin en sí de las propuestas formuladas: contribuir a elevar las capacidades de la gente para alcanzar los objetivos trazados. Analíticamente, se trata de una visión reduccionista que quita protagonismo a un momento crucial en la formulación de proyectos: la problematización reflexiva y crítica de la realidad vivida, paso que antecede a la formulación del problema.

Bajo este enfoque, aprender a leer la realidad desde una perspectiva crítica y problematizadora sería la principal necesidad formativa, al ser esta la base para definir, en primera instancia, qué se desea o requiere transformar. Conocimiento que ha de ir acompañado de la adopción del pensamiento lógico para la formulación de propuestas de intervención. Es decir, una vez definido el propósito común, usar el pensamiento para idear creativa y ordenadamente la cadena de acciones que deben emprenderse para disolver 0 mejorar sustancialmente los nudos críticos de las situaciones problemáticas (Aldana \& Reyes, 2004), incluyendo prever los recursos y los tiempos necesarios para llevar a cabo las transformaciones deseadas, acciones en conjunto equivalentes a la formulación de un proyecto.

En línea con lo anterior, una vez concebido el camino a seguir, en lo posible construido participativamente, ahora sí habría espacio para lo instrumental: plasmar lo ideado en los formatos dispuestos para la presentación formal de las propuestas con fines de cofinanciación, haciendo los ajustes a lugar. En este escenario, conocer los marcos y las formalidades con los que instituciones gubernamentales y no gubernamentales operan en su rol de entidades financiadoras o administradoras de recursos tendría sentido como necesidad formativa. Requerimiento condensado en saber adaptar las ideas generadas a las lógicas y solicitudes específicas de las entidades financiadoras como vía para la gestión de recursos. Pericia que ha de ir de la mano con saber reconocer cuál es la oferta institucional disponible y dónde hallar dicha información.

En resumen, se trata de una necesidad formativa que remite al encuentro dialógico entre el saber instrumental y el pensamiento crítico en torno a la propuesta de soluciones creativas a situaciones problemáticas. Aprender a problematizar la realidad, a observarla desde diferentes ángulos, a entenderla e interpretarla en sus distintas dimensiones, sería la base para posteriormente proponer, creativa y ordenadamente, los pasos requeridos para resolver los puntos críticos hallados. Aprendizaje que ha de complementarse con el conocimiento de las lógicas operativas de las entidades aportantes de recursos, incluyendo la actualización permanente sobre las políticas, normas institucionales y fuentes de acceso a recursos.

\section{Conclusiones y reflexiones finales}

Escuchar la voz de los extensionistas rurales, darles protagonismo en la definición de sus principales necesidades formativas, es una manera de complementar y ampliar lo sugerido sobre el tema desde la academia y la institucionalidad asociada al desarrollo agrícola y rural. Contribución que agrega al acervo actual sobre extensión rural direccionada a comunidades campesinas un importante referente experiencial, basado en las vivencias cotidianas de quienes, desde lo local, ejercen la labor de extensionistas rurales. Para el caso de estudio, las necesidades identificadas fueron: a) formación para la comunicación efectiva, necesidad basada en aprender cómo favorecer la generación de espacios para el diálogo principalmente horizontal entre extensionistas y agricultores, tras la búsqueda de objetivos comunes, b) formación para la práctica en contexto, necesidad fundada en aprender a conocer las especificadas del contexto social (en este caso campesino) en el cual tiene lugar la práctica extensionistas, y c) formación para la práctica interdisciplinaria, necesidad basada en aprender a sumar prácticas y saberes en torno a la resolución de circunstancias que, al ir más allá de lo únicamente técnico-productivo, ameritan ser intervenidas recurriendo a distintas disciplinas.

Son necesidades formativas con cabida diferencial (amplia, relativa, intermedia o limitada, según la valoración otorgada por el lector) en otros contextos de estudio, como sería 
el caso de la extensión pública o privada dirigida a grandes productores o de economía empresarial, la conducente a posicionar lógicas y modelos productivos particulares o la llevada a cabo por empresas privadas con fines principalmente comerciales, entre otros escenarios, que podrían, en su trasfondo, resultar útiles para cualificar la práctica extensionista en ámbitos como los mencionados, al adaptar lo dicho a sus especificidades (conocer las particularidades de la agricultura empresarial para actuar en contexto; involucrar a los contratantes del servicio privado de asistencia técnica en el diseño de las estrategias agroproductivas a sugerir, valorando sus perspectivas y conocimientos previos sobre el tema, por ejemplo).

En síntesis, se trata de diversas necesidades formativas, dialógicamente identificadas, a ser consideradas, a manera de sugerencia o recomendación constructiva, por quienes asumen la función social de formar profesionales para la extensión rural. En este sentido, los hallazgos constituyen un significativo insumo para los colectivos docentes y miembros de comités curriculares de programas de pregrado y posgrado afines al desarrollo y la extensión rural, interesados en perfeccionar los procesos de formación específica e integral de los futuros y actuales extensionistas. Necesidades a tenerse en cuenta en el diseño de propuestas de educación formal y no formal en extensión, esta vez con la oportunidad de estar basadas en el conocimiento derivado de la investigación social. En un primer momento, ajustar o complementar lo actualmente disponible (planes curriculares, programas de asignaturas y cursos) con lo localizadamente expuesto por los propios extensionistas sería un significativo paso hacia el mejoramiento permanente de su cualificación. Sugerencia que llama a adelantar trabajos similares en contextos socioculturales u geográficos distintos.

No obstante, concretar lo sugerido implica, sobre todo en escenarios de formación en ciencias agropecuarias, continuar ganando espacio para la formación sociohumanística, marco general en el que suele encuadrarse la formación en extensión y desarrollo rural. En el contexto de lo aquí discutido, lograr el equilibrio entre "lo social" y "lo técnico" sería un avance deseable y necesario en procura de la formación integral de los extensionistas. Ganancia que no solo ha de ser numérica (aumento en el número de créditos u horas), sino también en términos de concepción y perspectiva. Para el caso específico de las ciencias agropecuarias, concebir integralmente la producción agropecuaria y ganar conciencia de que esta es realizada por sujetos sociales con quienes los extensionistas y otros profesionales han de interactuar continuamente, contribuiría a alcanzar dicho equilibrio.

Mas, si la invitación permanente es a concebir una práctica extensionista en contexto, ¿qué tanta oportunidad de aproximarse a la realidad rural y agroproductiva se les da hoy a los discentes?

Pienso que a los profesionales que de una u otra forma tenemos que ver con lo rural nos falta por lo menos una asignatura que nos sumerja en el campo. En mi caso y el de mis compañeros, nosotros conocíamos los cultivos que posteriormente íbamos a transformar, pero no la vida del productor como tal. No teníamos ninguna relación con los agricultores que producían esos productos. Visitábamos los cultivos, casi siempre cultivos empresariales, sin tener relación con los productores. Yo pienso que es muy importante conocer algo de la realidad de las personas. Creo que no está bien llegar a campo sin conocer su realidad. (Extensionista No. 15).

Testimonios típicos como el anterior conllevan a plantear incógnitas sobre la capacidad de respuesta de las unidades académicas (programas y facultades, principalmente) a las necesidades expuestas por los extensionistas rurales: ¿es posible formarse para la práctica en contexto en el marco de un escenario formativo que poco interactúa con el entorno social?, ¿cómo formarse para la comunicación efectiva cuando se está sumido en un ambiente académico en el que mínimamente se dialoga?, ¿cómo formarse para la práctica integral en un entorno formativo usualmente concentrado en la cuestión técnico-productiva? Preguntas como estas invitan a pensar sobre cuáles han de ser las necesidades de ajuste de aquellos escenarios en los que comúnmente se forman quienes optan por ejercer la labor extensionista. 
Premisa final que convida a continuar problematizando la formación en extensión rural y a avanzar en el proceso de construcción permanente de una extensión cada vez más integral, puesta al servicio de las comunidades rurales y de la sociedad en su conjunto.

\section{Referencias bibliográficas}

Aldana, E., \& Reyes, A. (2004). Disolver problemas: criterios para formular proyectos sociales. Bogotá: Universidad de los Andes.

Alemany, C., \& Sevilla, E. (2007). ¿Vuelve la extensión rural? Reflexiones y propuestas agroecológicas vinculadas con el retorno y fortalecimiento de la extensión rural en América Latina. Realidad Económica, 227, 52-74.

Altieri, M. (1994). Bases agroecológicas para una producción agrícola sustentable. Agricultura Técnica (Chillán), 54(4), 371-386.

Altieri, M., \& Nicholls, C. (2010). Agroecología: potenciando la agricultura campesina para revertir el hambre y la inseguridad alimentaria en el mundo. Revista de Economia (Curitiba), 10(2), 62-74.

Amézaga, C., Rodríguez, D., Núñez, M., \& Herrera, D. (2013). Orientaciones estratégicas para el fortalecimiento de la gestión asociativa. San Salvador: IICA.

Attride, J. (2001). Thematic networks: an analytic tool for qualitative research. Qualitative Research, 1(3), 385-405.

Barrientos, M., \& Ryan, S. (2017). Relación entre extensión, educación y comunicación. Buenos Aires: UNICEN.

Bauman, Z. (1989). Modernidad y holocausto. Madrid: Sequitur.

Borsese, A., \& Esteban, S. (2005). Comunicación y lenguaje en el proceso de enseñanza-aprendizaje. Enseñanza de las Ciencias, 24(extra), 1-5.

Cáceres, D., Silvetti, F., Soto, G., \& Rebolledo, W. (1997). La adopción tecnológica en sistemas agropecuarios de pequeños productores. Agro Sur, 24(2), 123-135.

Camacho, P., Marlin, C., \& Zambrano, C. (2007). Elementos orientadores para la gestión de empresas asociactivas rurales-EMARs. Quito: RURALTER

Caporal, R., \& Dambrós, O. (2017). Extensão rural agroecológica: experiências e limites. Revista REDES, 22(2), 275-297.

Corporación Colombiana de Investigación Agropecuaria - CORPOICA. (2015). Ciencia, Tecnología e Innovación en el Sector Agropecuario. Diagnóstico: Misión para la Transformación del Campo. Bogotá: DNP.

De Aquino, S., \& Mengel, A. (2018, noviembre 25-30). A produção local de soluções tecnológicas para a agricultura familiar e o papel e o saber dos ferreiros. In X Congreso de la Asociación Latinoamericana de Sociología Rural. Montevideo, Uruguay: ALASRU-Universidad de la República.

De Souza, I. (2006). Agricultura familiar na dinâmica da pesquisa agropecuária. Brasília: Embrapa.

Delgado, F., \& Rist, S. (2016). Las ciencias desde la perspectiva del diálogo de saberes, la transdisciplinariedad y el diálogo intercientífico. In F. Delgado, \& S. Rist (Eds.), Ciencias, diálogo de saberes e interdisciplinariedad. Aporte teórico metodológico para la sustentabilidad alimentaria y el desarrollo (pp. 35-60). La Paz: AGRUCO-UMSS.

Diesel, V., Neumann, P., Silveira, P., \& Froehlich, M. (2006). Entendendo a diversidade e superando a fragmentação da ação extensionista. In H. Tommasino \& P. De Hegedüs (Orgs.), Extensión: reflexiones para la intervención en el medio urbano y rural (pp. 31-44). Montevideo: Universidad de la Republica, Montevideo.

Ferraro, C., \& Goldstein, E. (2011). Políticas de acceso al financiamiento para las pequeñas y medianas empresas en América Latina. Santiago de Chile: CEPAL-AECID.

Forero, J. (2003). Economía campesina y sistema alimentario en Colombia: aportes para la discusión sobre seguridad alimentaria. Bogotá: Pontificia Universidad Javeriana.

Gil, F., Rico, R., \& Sánchez-Manzanares, M. (2008). Eficacia de equipos de trabajo. Papeles del Psicólogo, 29(1), 25-31.

Gómez J., \& Elboj, C. (2001). El giro dialógico de las ciencias sociales: hacia la comprensión de una metodología dialógica. Acciones e Investigaciones Sociales, 12, 77-94. 
Lacey, H. (2015). A agroecologia: uma ilustração da fecundidade da pesquisa multiestratégica. Estudos Avançados, 29(83), 175-181.

Landini, F. (2011). Racionalidad económica campesina. Mundo Agrario, 12(23). Recuperado el 20 de agosto de 2020, de https://www.mundoagrario.unlp.edu.ar/article/view/225

Landini, F. (2015). Concepción de "extensión rural" de los extensionistas rurales argentinos que trabajan en el sistema público nacional con pequeños productores. Cuadernos de Desarrollo Rural, 12(75), 33-53.

Landini, F. (2021). Cambios en la comprensión del propio rol de extensionista a partir de la experiencia: un estudio latinoamericano. Revista de Economia e Sociologia Rural, 59(2), e224267. http://dx.doi.org/10.1590/1806-9479.2021.224267

Landini, F., Bianqui, V., \& Russo, M. (2013). Evaluación de un Proceso de Capacitación para Extensionistas Rurales Implementado en Paraguay. Revista de Economia e Sociologia Rural, 51(Supl. 1), s009-s030.

Lettelier, D., Dalmasso, C., \& Bernabé, E. (2016, noviembre 16-18). Metodología de sistematización de experiencias como herramienta de enseñanza para la formación en Extensión Rural. In $V$ Encuentro Latinoamericano de Metodología de las Ciencias. Mendoza, Argentina: UNLP-FaHCE.

Liebman, M. (1997). Sistemas de policultivos. In M. Altieri (Org.), Agroecología. Bases científicas para una agricultura sustentable (pp. 133-141). La Habana: CLADES.

López, N. (1998). Competitividad y administración agropecuaria. Agronomia Colombiana, 16(2), 204-210.

Machado, A. (2000). El papel de las organizaciones en el desarrollo rural. Buenos Aires: CLACSO.

Méndez, M. (2006). Los retos de la extensión ante una nueva y cambiante noción de lo rural. Revista Facultad Nacional de Agronomía, 59(2), 3407-3423.

Méndez, M. (2008). Integración del componente sociohumanístico en la formación de profesionales en ciencias agropecuarias: percepciones, propósitos y desafíos. Revista Facultad Nacional de Agronomía, 61(2), 4471-4479.

Méndez, M. (2015). Satisfacción con el empleo de extensionista rural: un estudio cualitativo en Caldas. Revista Ceres, 62(3), 241-250.

Méndez, M. (2016). El rol social de la extensión rural: enfoques para una intervención interdisciplinaria. Revista de Educação Técnica e Tecnológica em Ciências Agrícolas, 11(14), 32-49.

Méndez, M. (2020). Hacia una extensión rural fundada en el diálogo sinérgico de saberes: extensionistas y campesinos construyendo juntos. Revista REDES, 25(1), 257-274.

Menezes, F., \& Miranda, J. (2011). Um olhar ético e moral nas ações extensionistas. Revista de Ciências Humanas, 11(1), 139-150.

Moreno, F. (2015). La utilización de los materiales como estrategia del aprendizaje sensorial. Opción, 31(2), 772-789.

Muñoz, C. (2010). El rol de la metáfora léxica en la divulgación científica. Tabula Rasa, 13, 273-292.

Norgaard, R. (1994). Development betrayed: the end of progress and a coevolutionary revisiting of the future. London: Routledge.

Nussbaum, M. (2011). Sin fines de lucro. Buenos Aires: Katz.

Ramos, G. (2006). La formación humanística como componente de la formación integral del profesional universitario. Revista Educação em Questão, 13, 7-27.

Ringuelet, R., Cacivio, R., \& Rey, M. (2015). Situaciones problemáticas en los procesos de extensión rural. Revista Facultad de Agronomía, 114(esp. 1), 169-177.

Rodríguez, M., Salguero, Z., \& Ginebra, A. (2017). Las competencias laborales del cargo de agente de extensión agraria. Revista de la Facultad de Agronomía, 116(3), 39-44.

Russo, R. (2009). Capacidades y competencias del extensionista agropecuario y forestal en la globalización. Revista Comunicación, 18(2), 86-91.

Sánchez, C. (2016). Planificación financiera de empresas agropecuarias. Revista Científica Visión de futuro, 20(1), 209-227.

Santacoloma-Varón, L. (2015). Importancia de la economía campesina en los contextos contemporáneos: una mirada al caso colombiano. Entramando, 11(2), 38-50. 
Santos, L., \& Ramírez, E. (2016). De la contradicción entre la crisis de las profesiones agropecuarias y el potencial agropecuario colombiano. Revista Lebret, 8, 293-308.

Valderrama, M., \& Mondragón, H. (1998). Desarrollo y equidad con campesinos. Bogotá: IIICA-TM Editores.

Van der Ploeg, J. (2009). The new peasantries: struggles for autonomy and sustainability in an era of empire and globalization. Virginia: Earthscan.

Villegas, E. (2002). Análisis financiero en los agronegocios. Revista Mexicana de Agronegocios, 6(10), 337346.

Vizcaino, A., \& Otero, I. (2007). Enseñar-aprender para el desarrollo: la interdisciplinariedad como una alternativa de solución. Revista Pedagogía Universitaria, 12(2), 67-87.

Data de submissão: 20 de agosto de 2020.

Data de aceite: 31 de janeiro de 2021.

JEL: O13, Q16 